Active liver $X$ receptor signaling in phagocytes in multiple sclerosis lesions Peer-reviewed author version

MAILLEUX, Jo; VANMIERLO, Tim; BOGIE, Jeroen; WOUTERS, Elien; Lütjohann, Dieter; HENDRIKS, Jerome \& VAN HORSSEN, Jack (2018) Active liver $X$ receptor signaling in phagocytes in multiple sclerosis lesions. In: Multiple Sclerosis Journal, 24 (3), p. 279-289.

DOI: $10.1177 / 1352458517696595$

Handle: http://hdl.handle.net/1942/23716 


\section{ACTIVE LIVER X RECEPTOR SIGNALING IN MULTIPLE SCLEROSIS LESIONS}

\section{Abstract}

Objective: We sought to determine the LXR ligands present in human macrophages after myelin phagocytosis and whether LXRs are activated in MS lesions.

Methods: We used real-time quantitative PCR and immunohistochemistry to determine expression of LXRs and their response genes in human phagocytes after myelin phagocytosis and in active MS lesions. We used gas chromatographic/mass spectrometric analysis to determine LXR-activating oxysterols and cholesterol precursors present and formed in myelin and myelin-incubated cells, respectively.

Results: Myelin induced LXR response genes ABCA1 and ABCG1 in human monocyte-derived macrophages. In active MS lesions, we found that both gene expression and protein levels of ABCA1 and APOE are upregulated in foamy phagocytes. Moreover, we found that the LXR ligand 27-hydroxycholesterol $(270 H C)$ is significantly increased in human monocyte-derived macrophages after myelin uptake.

Conclusions: LXR response genes are upregulated in phagocytes present in active MS lesions, indicating that LXRs are activated in actively demyelinating phagocytes. In addition, we have shown that myelin contains LXR ligands and that $270 \mathrm{HC}$ is generated in human monocyte-derived macrophages after myelin processing. This suggests that LXRs in phagocytes in active MS lesions are activated at least partially by (oxy)sterols present in myelin and the generation thereof during myelin processing. 


\section{Introduction}

Multiple sclerosis (MS) is a chronic inflammatory, demyelinating, and neurodegenerative disease of the CNS (1). Acute inflammatory focal demyelination and the resulting neurodegeneration are among the most prominent pathological hallmarks of MS. Monocyte-derived macrophages and resident microglia secrete inflammatory and toxic mediators that negatively impact axonal and myelin integrity (2-4). Moreover, phagocytes contribute to MS lesion formation by phagocytosing myelin, apoptotic cells, and cellular debris. Although degeneration of myelin is considered detrimental, its clearance is essential prior to initiation of remyelination. Myelin debris contains proteins that inhibit both axonal re-growth and differentiation of oligodendrocyte precursor cells into mature oligodendrocytes during remyelination $(5,6)$. This emphasizes the cardinal role of phagocytes in stimulating CNS repair.

Cholesterol is the most abundant lipid within myelin (7) and plays a key role in myelination, illustrated by perturbed myelin formation in mice with defective cholesterol synthesis in the myelin-forming oligodendrocytes (8). Virtually all cholesterol within the CNS is synthesized in situ and a stable turnover is necessary to maintain cholesterol homeostasis (9). Breakdown of myelin results in a disturbed CNS cholesterol metabolism in MS patients, demonstrated by altered plasma levels of CNS-specific cholesterol metabolites 24(S)-hydroxycholesterol (24SOHC) and 27-hydroxycholesterol (27OHC) (10-12). Infiltrating macrophages and resident microglia phagocytose myelin leading to an increased uptake of cholesterol. This may lead to the formation of intracellular oxysterols. Oxysterols and other intermediates of the cholesterol biosynthetic pathway, such as desmosterol, are natural liver X receptor (LXR) ligands, fueling cellular sterol efflux (13). Both isoforms, LXRa and LXRß, are expressed in the CNS and immune cells $(14,15)$. When activated, LXRs heterodimerize with retinoid $x$ receptors (RXR). They regulate the expression of genes that participate in reverse cholesterol transport, such as ATP binding cassette transporter $(A B C) A 1, A B C G 1$, and apolipoprotein E (APOE). LXRs have also emerged as suppressors of inflammatory pathways in macrophages through SUMOylation $(16,17)$. Evidence is emerging that macrophages display an antiinflammatory phenotype upon myelin phagocytosis (18-20), suggesting that myelin contains functional LXR ligands or leads to the formation thereof. Recently, we demonstrated that myelin-derived lipids are able to activate LXRs in rat macrophages and skew these macrophages towards a less inflammatory phenotype in vitro (21). However, it is currently unknown if LXRs are activated in phagocytes in active MS lesions. We hypothesize that the uptake of myelin and the subsequent formation of LXR ligands, results in the activation of LXRs in phagocytes in active MS lesions.

We show that phagocytosis of myelin by human monocyte-derived macrophages leads to a significant induction of LXR response genes. In addition, we show that infiltrated macrophages and resident microglia 
express enhanced levels of LXRa, ABCA1 and APOE in active demyelinating lesions. Lastly, we show that the oxysterol $270 \mathrm{HC}$ is formed after myelin uptake and processing. The formation of this LXR ligand may explain the LXR activation seen in myelin-phagocytosing phagocytes in active demyelinating lesions. 


\section{Methods}

\section{Myelin Isolation}

Myelin was purified from human brain tissue by density-gradient centrifugation, as described previously (22). Protein concentration was determined using a BCA protein assay kit (Thermo Fisher Scientific, Erembodegem, Belgium). Endotoxin content was determined using a LAL Chromogenic endotoxin quantitation kit (Thermo Fisher). The isolated myelin contained a negligible amount of endotoxin $(<1.8 \times 10-$ $3 \mathrm{pg} / \mu \mathrm{g}$ myelin).

\section{Cell culture}

Human peripheral blood mononuclear cells (PBMCs) were obtained from consented healthy donors using Ficoll (Cedarlane Labs, Canada) density gradient. Monocytes were isolated from the PBMCs by anti-CD14 EasySep microbeads (Stemcell technologies, Grenoble, France) according to manufacturer's instructions. Monocytes (1x106 cells/ml) were cultured in 24-well plates (Greiner Bio-one) in macrophage differentiation medium (RPMI (Lonza, Vervier, Belgium), supplemented with $10 \% \mathrm{v} / \mathrm{v}$ normal fetal calf serum (Life Technologies, Ghent, Belgium) and $50 \mathrm{U} / \mathrm{ml}$ v/v penicillin-streptomycin (Sigma-Aldrich, Bornem, Belgium)), at $37^{\circ} \mathrm{C}, 5 \% \mathrm{CO} 2$. Monocytes matured into macrophages in the course of 7 days. For experiments, myelin $(100 \mu \mathrm{g} / \mathrm{ml})$ was added to macrophages for 24 hours preceding mRNA extraction.

\section{Autopsy material}

Brain tissue samples were obtained from the Netherlands Brain Bank. All patients, healthy controls or their next of kin, had given informed consent for autopsy and use of brain tissue for research purposes. Active lesions were immunohistochemically characterized as lesions with abundant immune cell infiltrates and extensive myelin loss (table 1 ).

Table 1 Brain tissue characteristics. (NDC; non demented controls, RR-MS; relapse remitting MS, PPMS; primary progressive MS; SP-MS; secondary progressive MS). Cases marked* were used for IHC, cases marked + were used for qPCR.

\begin{tabular}{llllll}
\hline $\mathbf{N r}$ & Autopsy & Diagnosis & Gender & Age & Type MS \\
\hline 1 & $\mathbf{5 9 4 - 1 6 3 ^ { + }}$ & NDC & Female & $74 y$ & - \\
\hline 2 & $\mathbf{S 9 5 - 0 1 9}^{+}$ & NDC & Male & $54 y$ & - \\
\hline 3 & $\mathbf{S 9 6 - 3 7 3}^{+}$ & NDC & Male & $70 y$ & - \\
\hline 4 & $\mathbf{S 0 1 - 0 1 6}^{+}$ & NDC & Female & $64 y$ & - \\
\hline 5 & $\mathbf{S 0 1 - 1 7 4}^{+}$ & NDC & Female & $47 y$ & - \\
\hline 6 & $\mathbf{S 9 7 - 1 3 1}^{+}$ & NDC & Female & $65 y$ & - \\
\hline 7 & $\mathbf{S 0 1 - 5 8}^{+}$ & MS & Female & $48 y$ & RR-MS \\
\hline
\end{tabular}




\begin{tabular}{llllll}
8 & $\mathbf{S 0 1 - 2 9 8}^{+}$ & MS & Female & $53 y$ & RR-MS \\
\hline 9 & $\mathbf{S 0 3 - 1 4 2}^{+}$ & MS & Male & $53 y$ & PP-MS \\
\hline 10 & $\mathbf{S 0 4 - 0 4 5}^{+}$ & MS & Female & $41 y$ & RR-MS \\
\hline 11 & $\mathbf{S 9 9 - 2 6 1 *}^{*}$ & MS & Female & $38 y$ & RR-MS \\
\hline 12 & $\mathbf{S 0 7 - 2 6 9 *}_{13}^{*}$ & MS & Male & $41 y$ & PP-MS \\
\hline 14 & $\mathbf{S 0 7 - 3 1 4}^{+}$ & MS & Female & $66 y$ & SP-MS \\
\hline 15 & $\mathbf{S 0 9 - 1 5 2 *}$ & MS & Male & $50 y$ & Unknown \\
\hline
\end{tabular}

\section{Quantitative PCR}

Total RNA was prepared using the RNeasy mini kit (Qiagen, Venlo, The Netherlands) according to the manufacturer's instructions with the following modification: Qiazol lysis reagent (Qiagen) with $1 \% \beta-$ mercaptoethanol (Sigma-Aldrich) was used as lysis buffer. RNA concentration and purity was determined using a Nanodrop spectrophotometer (Isogen Life science). RNA was converted to cDNA using qScript cDNA SuperMix (Quanta Biosciences, Boston, USA) according to the manufacturer's instructions. Quantitative PCR (qPCR) was conducted on a StepOnePlus ${ }^{\mathrm{TM}}$ Real-Time PCR system (Applied biosystems, Ghent, Belgium). The SYBR green master mix (Applied biosystems), $10 \mu \mathrm{M}$ of forward and reverse primers, nuclease free water and $12.5 \mathrm{ng}$ template cDNA in a total reaction volume of $10 \mu \mathrm{l}$. Relative quantification of gene expression was accomplished by using the comparative Ct method. Data were normalized to the most stable reference genes. Primers were chosen according to literature or designed using Primer-express (http://www.ncbi.nlm.nih.gov/tools/primer-blast). Details of primers used are shown in table 2.

Table 2 Primers used to measure the relative expression of different human genes.

\begin{tabular}{|c|c|c|c|}
\hline Gene & Sequence $\left(5^{\prime}-3^{\prime}\right)$ & Product size & Accession number \\
\hline LXRa forward & CGCACTACATCTGCCACAGT & \multirow{2}{*}{159 bp } & \multirow{2}{*}{ NM_001251935.1 } \\
\hline LXRa reverse & CTTGCCGCTTCAGTTTCTTC & & \\
\hline LXRß forward & CAGCAGCAGGAGTCACAGTC & \multirow[b]{2}{*}{157 bp } & \multirow[b]{2}{*}{ NM_001256647.1 } \\
\hline LXR $\beta$ reverse & GTTCTTGAGCCGCTGTTAGC & & \\
\hline ABCA1 forward & AACGAGACTAACCAGGCAATC & \multirow[b]{2}{*}{148 bp } & \multirow[b]{2}{*}{ NM_005502.3 } \\
\hline ABCA1 reverse & ACACAATACCAGCCCAGAAC & & \\
\hline ABCG1 forward & CCAGAAGTCGGAGGCCATC & \multirow[b]{2}{*}{$63 \mathrm{bp}$} & \multirow[b]{2}{*}{ NM_207629.1 } \\
\hline ABCG1 reverse & AAGTCCAGGTACAGCTTGGCA & & \\
\hline APOE forward & ACTGGGTCGCTITTGGGATT & \multirow[b]{2}{*}{$64 \mathrm{bp}$} & \multirow[b]{2}{*}{ NM_000041.2 } \\
\hline APOE reverse & СTCСTCСTGCACСTGCTCA & & \\
\hline
\end{tabular}




\begin{tabular}{|c|c|c|c|}
\hline TBP forward & TATAATCCCAAGCGGTTTGC & \multirow[b]{2}{*}{$170 \mathrm{bp}$} & \multirow[b]{2}{*}{ NM_003194.4 } \\
\hline TBP reverse & GCTGGAAAACCCAACTTCTG & & \\
\hline CYCA forward & AGACTGAGTGGTTGGATGGC & \multirow[b]{2}{*}{$142 \mathrm{bp}$} & \multirow[b]{2}{*}{ NM_021130.3 } \\
\hline CYCA reverse & TCGAGTTGTCCACAGTCAGC & & \\
\hline YWHAZ forward & CTTGACATTGTGGACATCGG & \multirow[b]{2}{*}{$145 \mathrm{bp}$} & \multirow[b]{2}{*}{ NM_003406.3 } \\
\hline YWHAZ reverse & TATTTGTGGGACAGCATGGA & & \\
\hline Rpl13a forward & AAGTTGAAGTACCTGGCTTTCC & \multirow[b]{2}{*}{$171 \mathrm{bp}$} & \multirow[b]{2}{*}{ NM_012423.3 } \\
\hline Rpl13a reverse & GCCGTCAAACACCTTGAGAC & & \\
\hline
\end{tabular}

\section{Immunohistochemistry}

Snap-frozen brain tissue was sectioned at $5 \mu \mathrm{m}$ and stained for proteolipid protein (PLP, 1:500, MCA839G, AbD Serotec, Temse, Belgium) and HLA-DR (biotin-labeled clone LN3, eBioscience; 13-9956) to determine MS lesion type. All antibodies were diluted in phosphate buffered saline (PBS) with $1 \%$ bovine serum albumin (BSA, Sigma-Aldrich).

For DAB-stainings, sections were air-dried and fixed in acetone for 10 minutes. Endogenous peroxidase activity was quenched by incubating the slides in $0.3 \%$ hydrogen peroxide. Sections of each tissue sample were incubated either with goat anti-LXRa (1:200, kindly provided by Prof. Dr. Gustafsson), goat anti-LXR (1:200, kindly provided by Prof. Dr. Gustafsson), rabbit anti-ABCA1 (1:200, NB400-105, Novus biologicals, Denver, USA), rabbit anti-APOE (1:200, NBP1-19807, Novus biologicals), or rabbit anti-LDLR (1:400, NB110-57162, Novus biologicals) for 60 minutes at room temperature. Then, slides were incubated with EnVision+ Dual Link System-HRP (DAKO, Heverlee, Belgium) for 30 minutes or with HRP labeled rabbit anti-goat (1:100, DAKO) for 60 minutes at room temperature. 3, 3'-diaminobenzidine (DAB+, DAKO) was used as chromogen. Sections were thoroughly washed with PBS between each incubation step. After a short rinse in tap water, sections were incubated with hematoxylin for 1 minute and extensively washed with tap water for 10 minutes. Sections were dehydrated with ethanol followed by xylene and mounted with Entellan (Merck).

For immunofluorescence staining, sections were air-dried and fixed in acetone for 10 minutes. Non-specific binding was blocked using 1\% BSA in PBS for 30 minutes. Sections of each tissue sample were incubated either with anti-LXRa, anti-LXRß, anti-ABCA1, anti-APOE or anti-LDLR overnight at $4^{\circ} \mathrm{C}$ followed by incubation with Alexa-488-labeled donkey anti-goat (1:150, Molecular Probes) or Alexa-488-labeled goat anti-rabbit (1:400, Molecular Probes). To visualize HLA-DR positive macrophages, an antibody against HLADR (biotin-labeled clone LN3, eBioscience; 13-9956) was subsequently applied for 60 minutes. HLA-DR 
slides were further incubated with Alexa-555-labeled streptavidin (1:400, Molecular Probes). Analysis was carried out using a Nikon eclipse 80i microscope and NIS Elements BR 4.20 software (Nikon).

\section{Oil red $O$ staining}

Myelin treated human monocyte-derived macrophages were fixed using 4\% paraformaldehyde (SigmaAldrich) for 20 min. Next, cells were stained with oil red O (ORO) (Sigma-Aldrich) for 10 min and exhaustively rinsed with water. In order to quantify intracellular myelin levels, $1 \mathrm{ml}$ of isopropyl alcohol was added to the stained culture dish, the extracted dye was immediately removed by gentle pipetting and its absorbance determined spectrophotometrically at $510 \mathrm{~nm}$. To delineate active MS lesion tissue for qPCR, cryosections (unfixed) were stained with ORO for 10 min and exhaustively rinsed with water. Next, a hematoxylin counterstain was applied and cryosections were subsequently mounted.

\section{Sterol analysis}

Human monocyte-derived macrophages were treated with or without $100 \mu \mathrm{g} / \mathrm{ml}$ human myelin for 24 hours. Next, the medium containing myelin was collected and stored for further analysis, while cells were incubated for another four days in fresh medium. Cells were then lysed in $1 \mathrm{M} \mathrm{NaOH}$ (in $80 \%$ ethanol) and sterols were subsequently extracted from cell lysates and medium using chloroform-methanol (2:1). Sterol levels were determined by gas chromatograph mass spectrometer (GC/MS) as described previously (23, 24). The change in amount of sterols was determined by subtracting (condition with myelin) from (condition no myelin), and subsequently dividing it by the amount of sterols present in pure myelin after normalizing with the plant sterol campesterol, which is not metabolized by mammalian cells (25). A flow diagram of the experimental design is included in figure $4 \mathrm{~B}$.

\section{Statistical Analysis}

Data were statistically analyzed with GraphPad Prism 5 for windows and are reported as mean values \pm standard error (SEM). D'Agostino and Pearson omnibus normality test was used to test normal distribution. An analysis of variances (ANOVA) or two-tailed unpaired student t-test (with Welch's correction if necessary) was used for normally distributed data sets (t(df); P). The Mann-Whitney U analysis (MWU, n1, $\mathrm{n} 2$; P) was used for data sets which did not pass normality. $* \mathrm{P}<0,05, * * \mathrm{P}<0,01$ and $* * * \mathrm{P}<0,001$. 


\section{Results}

Induction of LXR response gene expression in human monocyte-derived macrophages after myelin uptake

To investigate if LXRs in human monocyte-derived macrophages are activated after myelin uptake, we determined the expression of well-known LXR downstream targets by quantitative real-time PCR (qPCR) analysis. mRNA expression of the autoregulated LXRa and LXR response genes ABCA1 and ABCG1 was increased in myelin-treated cells, compared to non-treated cells, whereas LXR $\beta$ and APOE mRNA levels remained unchanged (fig. 1). The Ct value of LXRa in unstimulated cells is lower than LXRß (LXRa:17.90 vs. $L X R \beta: 26.38)$. The threshold cycle is inversely proportional to the expression level indicating that $L X R a$ mRNA expression is $\sim 250$ times higher than LXRß in human macrophages (fig. S1). This indicates that LXRa appears to be the dominant driver behind LXR signaling in macrophages.

A

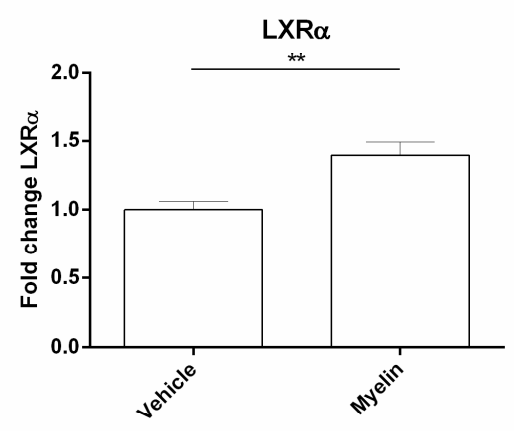

D

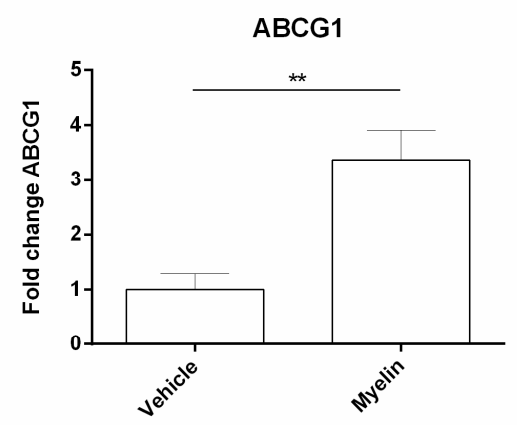

B

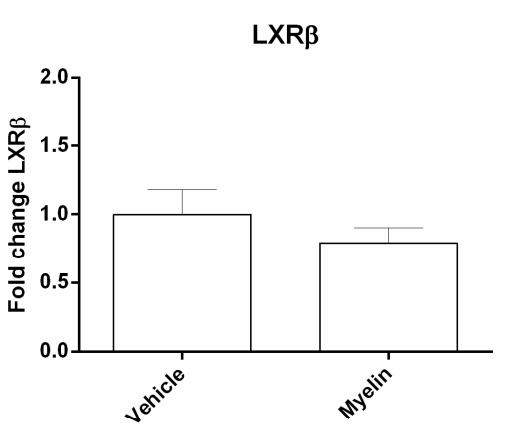

E

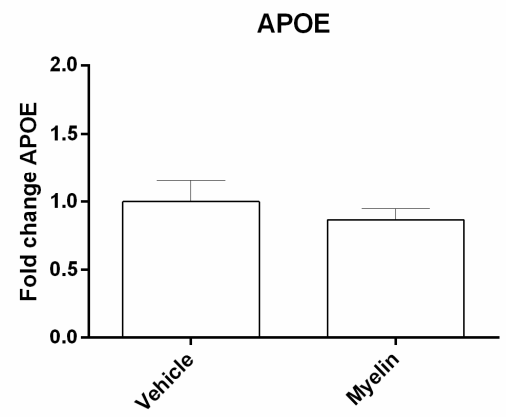

C

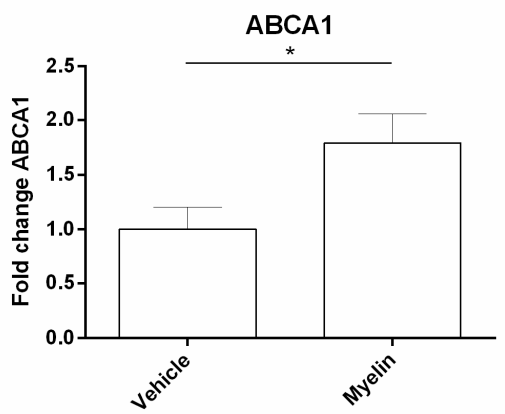

$\mathbf{F}$

\begin{tabular}{c|rr} 
Sample & LXRa $(C t)$ & LXRß $(C t)$ \\
\hline 1 & 17,9242 & 26,5524 \\
2 & 16,9822 & 25,9581 \\
3 & 17,2364 & 25,734 \\
4 & 18,4959 & 27,0704 \\
5 & 18,9064 & 26,5815 \\
Average & $\mathbf{1 7 , 9 0 9}$ & $\mathbf{2 6 , 3 7 9 3}$
\end{tabular}

Figure 1 LXR activation after myelin phagocytosis in human monocyte-derived macrophages.

Comparison of fold changes of LXRs and their response genes between untreated $(n=11)$ and myelintreated $(100 \mu \mathrm{g} / \mathrm{ml})$ human monocyte-derived macrophages $(n=11)$. Relative quantification of LXRa $(t(20)=3.516 ; p<0.003)(A), \operatorname{LXR\beta }(B), A B C A 1(M W U=29 ; 11 ; 11 ; p<0.039)(C), A B C G 1(M W U=8 ; 11 ;$ $11 ; p<0.001)(D)$ and APOE $(E)$ was accomplished by using comparative Ct method. Data were normalized 
to the most stable reference genes, determined by Genorm (TBP and CYCA).Induction of LXR response gene expression in active MS lesions

To determine whether LXRs are activated in MS lesions, gene expression levels of LXRs and downstream targets were determined (fig 2). RNA was isolated from regions accommodating lipid-containing macrophages and microglia, determined by Oil Red O (ORO) staining. mRNA expression of LXRa and its response genes $A B C A 1$ and $A P O E$ were increased in active lesions, compared to white matter tissue from non-neurological controls (fig. 2A, C, E). Moreover, LXRß mRNA expression was significantly reduced in active MS lesions, whereas the expression of ABCG1 remained unchanged. These results show LXR response genes are upregulated in active demyelinating MS lesions.

A

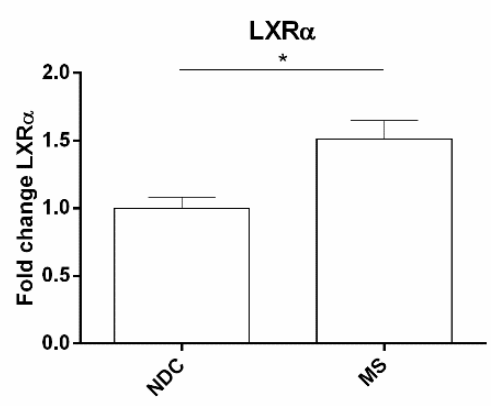

D

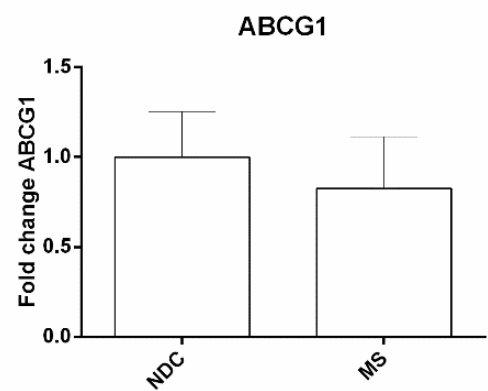

B

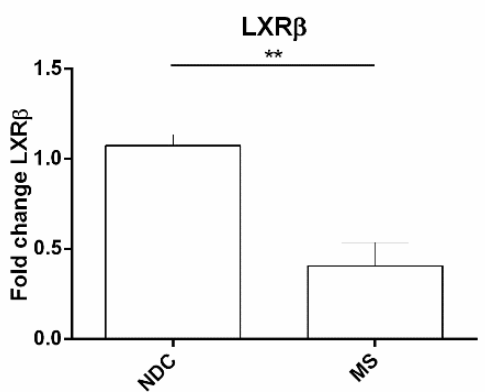

E

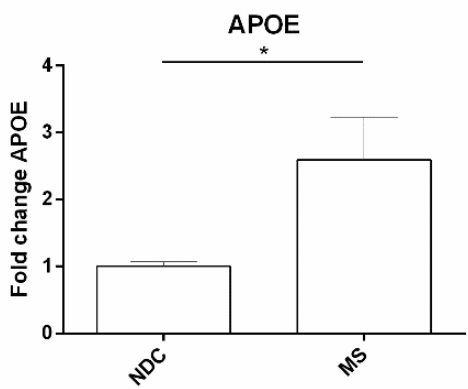

C

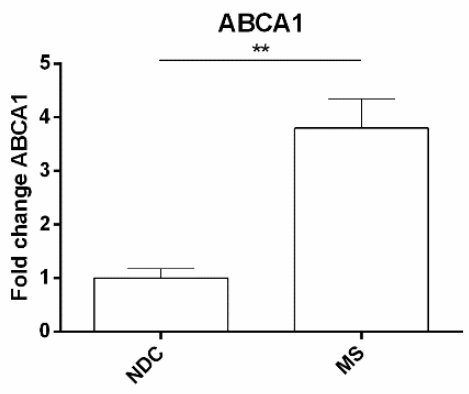

Figure 2 Active LXR signaling in MS lesions. Comparison of fold changes of LXRs and their response genes between non-demented controls $(n=6$, see table 1 nr:1-6) and active MS lesions ( $n=5$, see table 1 $\mathrm{nr}: 7-11)$. Relative quantification of $\operatorname{LXRa}(M W U=6 ; 6 ; 5 ; p<0.012)(A), \operatorname{LXR\beta }(t(11)=3.620 ; p<0.004)(B)$, ABCA1 $(t(12)=4.293 ; p<0.001) C), A B C G 1(D)$ and APOE (MWU=9; 6; 5; p<0.036) (E) was accomplished by using comparative Ct method. Data were normalized to the most stable reference genes, determined by Genorm (YWHAZ and Rpl13a). NDC; non-demented controls. 
To determine whether LXR response genes are also induced at protein level in MS lesions and to establish the cellular location of this induction, we determined the expression of LXRs and LXR response genes in active MS lesions by immunohistochemistry. For this purpose, we selected active demyelinating MS lesions of four MS cases (table 1). Identification of active lesions was based on immunohistochemical analysis of phagocytes (anti-HLA-DR) and proteolipid protein (PLP). Active lesions are characterized by extensive myelin loss and abundant myelin-containing phagocytic macrophages and microglia (fig. 3A-C). We found that the LXR response genes ABCA1, APOE, LXRa, and LXR $\beta$ are abundantly expressed in active lesions (fig. 3D-E, G-H, J-K, and M-N). Double immunofluorescence stainings revealed that ABCA1, APOE, and LXRa/ $\beta$ were highly expressed in HLA-DR+ cells with a foamy appearance (fig. 3F, I, L, O) suggesting that LXRs are activated in phagocytes in active MS lesions. 

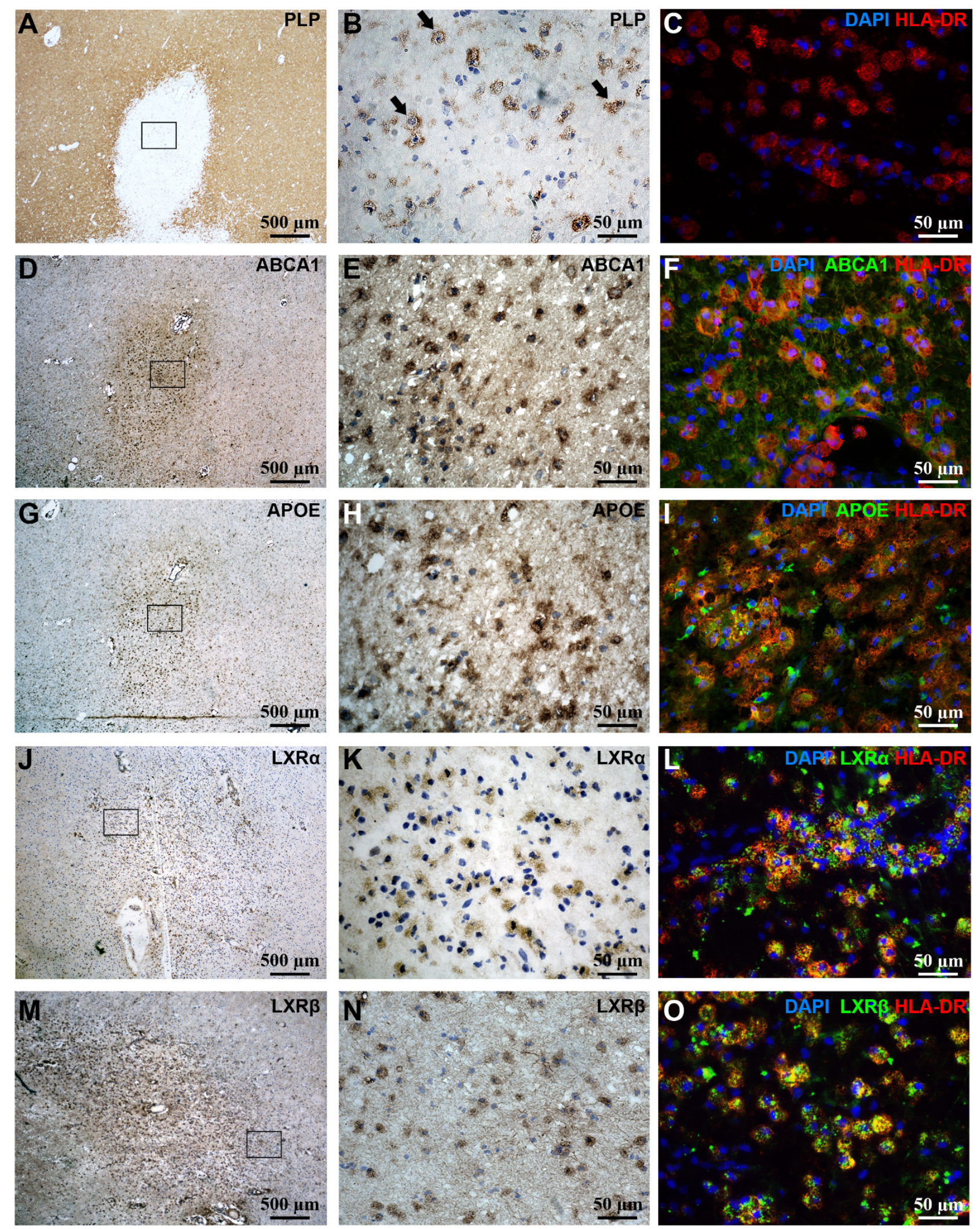

Figure 3 LXRs are activated in HLA-DR+ phagocytes in active MS lesions. Extensive demyelination and PLP+ phagocytic cells (black arrows) are observed in active MS lesions (A, B: proteolipid protein). Figure B represents a magnification of the outlined square in A. Active lesions are characterized by infiltrating macrophages and microglia expressing HLA-DR (C). ABCA1 (D, E), APOE (G, H), LXRa (J, K), and $\operatorname{LXR} \beta(M, N)$ were abundantly expressed by phagocytes. Figure $E, H$ and $K$ represent a magnification 
of the outlined square in $D, G$ and $J$ respectively. ABCA1 (F), APOE (I) and LXRa/ $\beta$ (L/O) were strongly expressed by HLA-DR+ phagocytes in active lesions. Original magnifications: A, D, G, J and M: 4x; B, C, E, F, H, I, K, L, N and O: 40x.

\section{7-hydroxycholesterol is formed in human monocyte-derived macrophages after myelin} ingestion

After myelin is phagocytosed, it is degraded inside the cell (fig. 4A). Myelin, visualized by ORO-staining, is rapidly phagocytosed and intracellular levels remain stable until day four. At day five a decline can be seen, indicating processing of myelin and possibly the release of cholesterol oxidation products. Using GC/MS we determined cellular oxysterol levels in human macrophages, treated with or without myelin incubated for five days. We compared these levels with the levels present in human myelin (fig. 4B-C). We found that while levels of cholesterol and the LXR ligand desmosterol were decreased after five days of myelin ingestion, $27 \mathrm{OHC}$, but not $240 \mathrm{HC}$ levels, were significantly increased in macrophages.

A
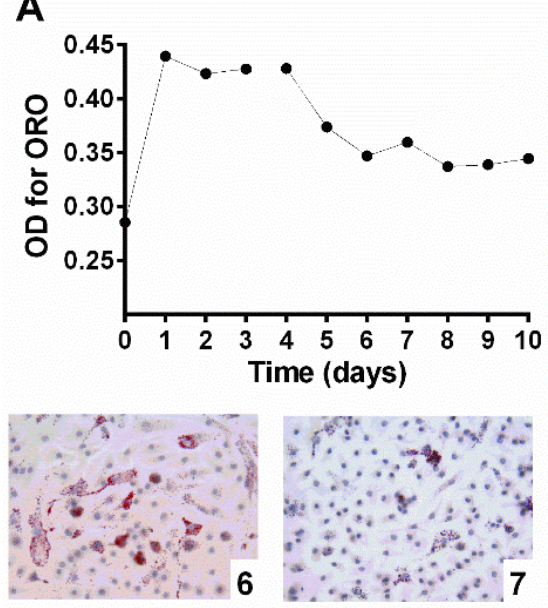
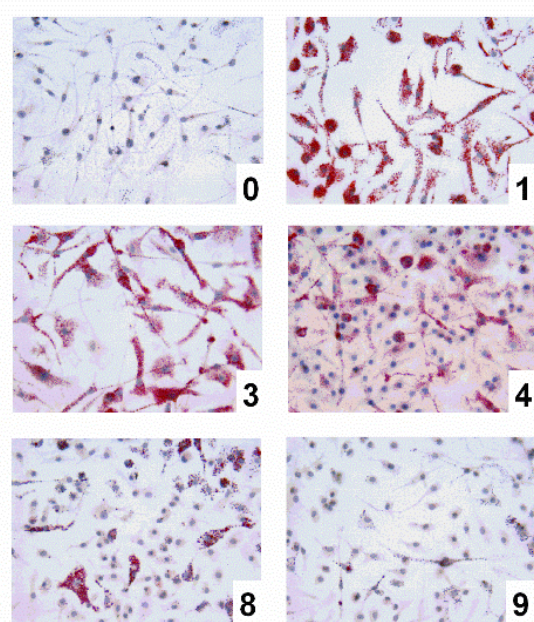
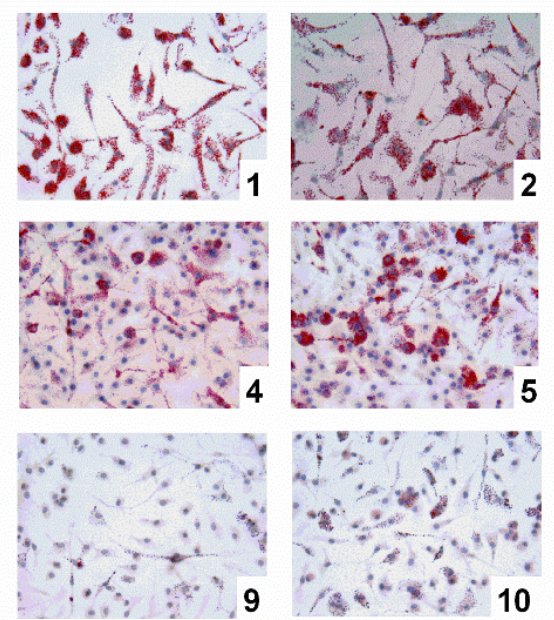

B
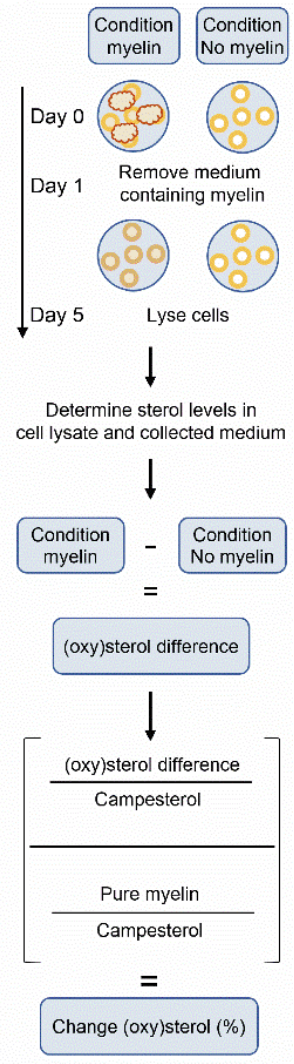

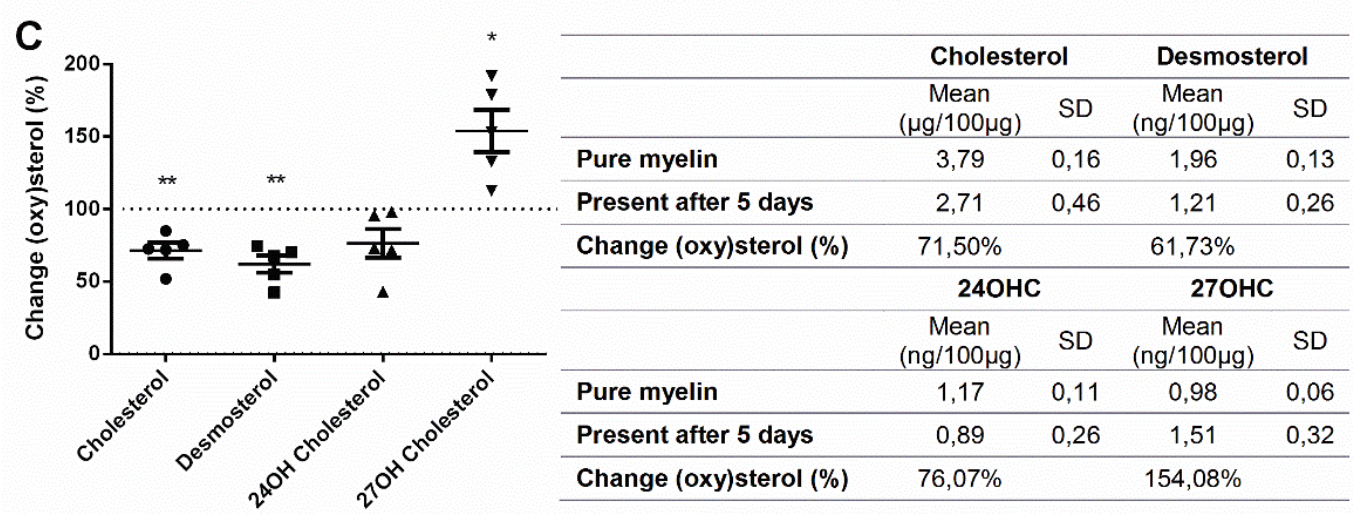

Figure 4 Degradation of myelin and the formation of $270 H C$. A) Monocyte-derived macrophages (0) were treated with $100 \mu \mathrm{g} / \mathrm{ml}$ myelin (24h) after which the unbound myelin was washed away and incubated for 10 consecutive days (1-10). Intracellular myelin was visualized by ORO staining. Quantification was performed by determining the extracted dye. B) Flow diagram of the experimental design. C) Human monocyte-derived macrophages were treated with $100 \mu \mathrm{g} / \mathrm{ml}$ myelin for $24 \mathrm{~h}$, after which the unbound 
myelin was washed away and cells incubated for another four days. Next, cholesterol, desmosterol and oxysterol levels were determined in the samples using GC/MS. Sterols were normalized using the plant sterol campesterol which is not metabolized. Relative levels of cholesterol $(t(4)=5.318 ; p<0.006, \bullet)$, desmosterol $(\mathrm{t}(4)=6.503 ; \mathrm{p}<0.003, \boldsymbol{m}), 24 \mathrm{OH}(\boldsymbol{\Delta})$ and $270 \mathrm{H}(\mathrm{t}(4)=3.720 ; \mathrm{p}<0.021, \boldsymbol{\nabla})$ are shown $(\mathrm{n}=5)$.

\section{7-hydroxycholesterol induces LXR response gene expression in human monocyte-derived macrophages}

We calculated that the concentration of $270 \mathrm{HC}$ present per $1 \times 106$ macrophages after five days of myelin incubation is around $5 \mu \mathrm{M}$. Therefore, we treated human monocyte-derived macrophages with $27 \mathrm{OHC}$ to determine whether the level of $270 \mathrm{HC}$ induced in myelin phagocytosing macrophages is sufficient to induce LXR response gene expression (fig. 5). The expression of $L X R a(M W U=0 ; 5 ; 5 ; p<0.008)$ and its response genes $A B C A 1(M W U=1 ; 5 ; 5 ; p<0.016)$ and $A B C G 1(M W U=0 ; 5 ; 5 ; p<0.008)$ was significantly increased in 27OHC-treated cells, compared to non-treated cells whereas LXR $\beta$ and APOE mRNA expression remained unchanged. These results suggest that the amount of $270 \mathrm{HC}$ present in vitro after myelin phagocytosis activates LXRs.

A

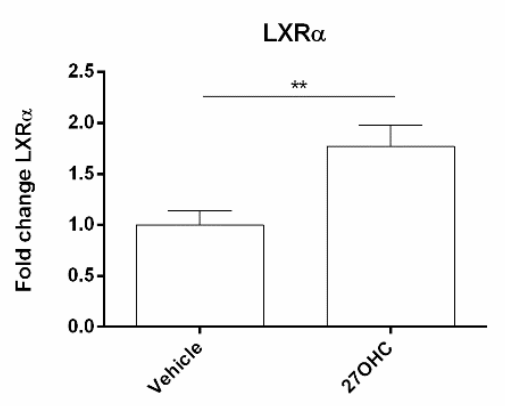

D

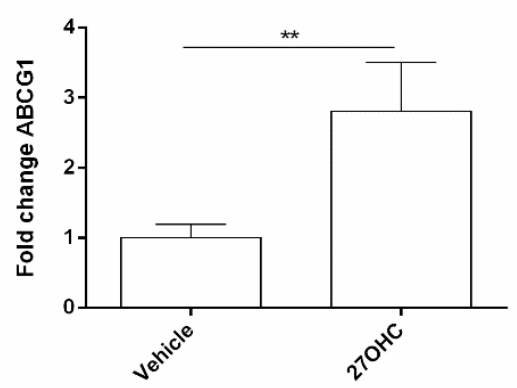

B

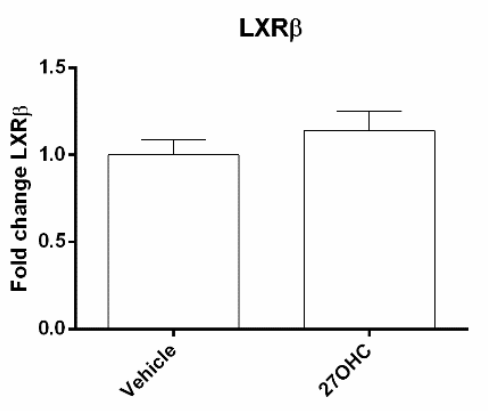

$\mathbf{E}$

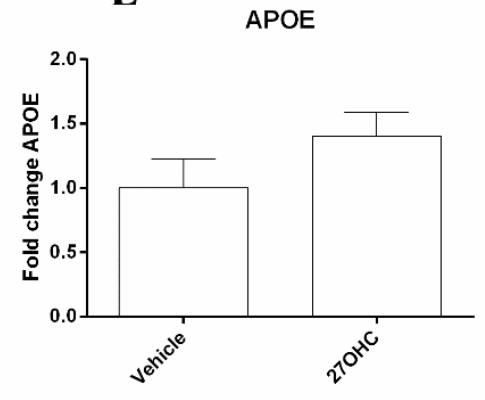

C

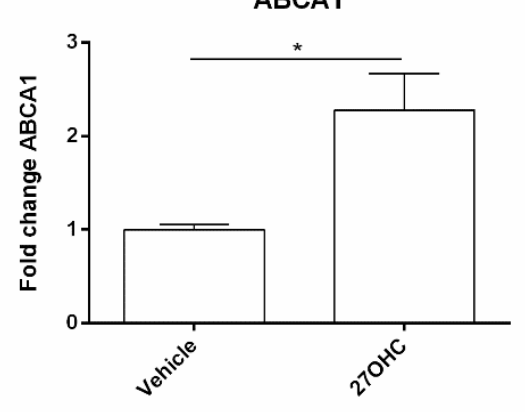

Figure 5 LXR activation after $\mathbf{2 7 0 H C}$ treatment. Comparison of fold changes between untreated $(n=5)$ and $270 \mathrm{OC}$-treated $(5 \mu \mathrm{M})$ human monocyte-derived macrophages $(n=5)$ for $24 \mathrm{~h}$. Relative quantification of LXRa (A), LXRß (B), ABCA1 (C), ABCG1 (D) and APOE (E) was accomplished by using comparative Ct method. Data were normalized to the most stable reference genes, determined by Genorm (YWHAZ and RPL13a). 


\section{Discussion}

In this study, we show that LXRs and their downstream targets are induced in myelin-laden macrophages in active demyelinating MS lesions, indicative of LXR activation. In active lesions, macrophages and activated microglia contribute to lesion progression by phagocytosing large quantities of myelin. Conceivably, upon myelin processing, LXR ligands are released leading to the upregulation and activation of LXRs, and their response genes. LXRs are master regulators of cholesterol metabolism and inflammatory responses and are critical for brain homeostasis. By activation of LXRs, reverse cholesterol transport is induced which protects myelin-phagocytosing cells from elevated intracellular free cholesterol and oxysterol-induced toxicity (26). In the brain, LXRß is ubiquitously expressed, whereas LXRa expression is largely restricted to infiltrating macrophages and microglia $(14,15)$.

We found LXRa expression in active lesions to be mainly present in HLA-DR ${ }^{+}$cells. Likewise, LXR $\beta$ expression was observed in HLA-DR+ cells and in perilesional astrocytes (data not shown). Though both isoforms are expressed by phagocytes, it appears that LXRa, at least at mRNA level, is the dominant driver behind LXR signaling in macrophages (27) which is also evidenced by our in vitro data (fig. 1). However, it is likely that due to translational and/or post-translational regulation the protein expression ratio differs from the gene expression ratio. In line herewith, we show that the difference in the expression level of LXRa and LXRß is less clear on protein level in acute MS lesions (fig. 3). Microarray analysis of myelinladen rat macrophages showed that the expression of LXR response gene ABCA1 was significantly upregulated compared to non-myelin treated cells (21). In concordance, we found that $A B C A 1$ is induced in both myelin-treated human monocyte-derived macrophages and active MS lesions. In summary, based on the observation that downstream targets of LXRs are highly upregulated in myelin-laden phagocytes it is likely that LXRs are activated upon myelin digestion in active MS lesions.

Myelin contains various lipids and oxysterols that can influence macrophage function. Recently, we demonstrated that myelin phagocytosis alters macrophage phenotype in rat macrophages through activation of LXRs (21). Therefore, we hypothesized that the uptake of myelin and the subsequent formation of LXR ligands results in the activation of LXRs in phagocytes in active MS lesions. We measured (oxy)sterols present in myelin such as desmosterol, $24 \mathrm{SOHC}$ and $27 \mathrm{OHC}$ that are known to potently activate LXRs (28-31). After myelin phagocytosis, myelin is processed by the phagocyte, possibly generating cholesterol oxidation products, such as oxysterols. We found that five days after uptake of myelin by macrophages, intracellular myelin levels start to decrease and the levels of the cholesterol metabolite $27 \mathrm{OHC}$, but not $24 \mathrm{SOHC}$ and desmosterol, are significantly elevated. Taken together, these results indicate 
that LXR ligands are present in human myelin, and that after myelin processing, $270 \mathrm{HC}$ is one of the major LXR ligands that is formed.

The amount of $270 \mathrm{HC}$ present in vitro in macrophages after myelin phagocytosis activates both LXRisoforms with a higher affinity for LXRa $(29,32)$. Exposing macrophages to $270 H C$ resulted, very similarly to myelin, in upregulation of $L X R a$, and LXR-response genes $A B C A 1$ and $A B C G 1$. Based on these data, we speculate that initially LXRs are activated by ligands present in myelin (e.g. 24SOHC, $270 \mathrm{HC}$ and desmosterol), while during later stages also LXR ligands that are generated after myelin processing contribute to LXR activation.

Collectively, based on the observation that downstream targets of LXRs are upregulated in myelin-laden phagocytes in active MS lesions it is conceivable that LXRs are activated by ligands present in myelin. Moreover, $270 \mathrm{HC}$ is the major LXR ligand that is formed after myelin processing and the levels of $270 \mathrm{HC}$ that are generated are capable of upregulating LXR downstream targets in phagocytes indicating that $270 \mathrm{HC}$ is, at least in part, responsible for activation of LXRs in MS lesions. Although myelin is very stable in content, it cannot be excluded that differences in myelin composition between and within individuals (e.g. different anatomical CNS regions) may modulate the conversion of cholesterol to $270 H C$. LXRs are of great importance in regulating cholesterol homeostasis and protect cells from elevated intracellular free cholesterol and oxysterol-induced toxicity (26). More importantly, LXRs have been shown to inhibit inflammatory gene expression and stimulate remyelination $(16,17,33,34)$ underscoring their significance as important regulators in MS pathogenesis. This notion is highlighted in a recent study in which a mutation was found in the LXRa gene in familial MS (35). The clinical disease progression and severity for mutant LXRa carriers is evidenced by its rapid progression and severity. In this study, the authors suggest that the p.Arg415GIn mutation, located in the ligand binding domain, prevents LXR activation by abrogating heterodimerization between LXR and RXR, resulting in loss of function. Because of the oxysterols present in myelin and the formation of cholesterol oxidation products, it is likely that myelin-laden phagocytes in MS are at least in part modulated by a myelin-mediated activation of LXRs, providing an intriguing hypothesis for the LXR-controlled self-limiting nature of MS lesion development. 


\section{Acknowledgements}

We are grateful to all individuals who participated in this study. We thank A. Kerksiek for the sterol analysis and K. Wauterickx for technical assistance. This work was supported by Agentschap voor Innovatie van Wetenschap en Technologie (IWT) (Grant 131086) and Fonds voor Wetenschappelijk Onderzoek (FWO) (Grants 1506916N \& 1512314N and 1264114N).

\section{References}

1. Bar-Or A, Oliveira EM, Anderson DE, Hafler DA. Molecular pathogenesis of multiple sclerosis. J Neuroimmunol. 1999;100(1-2):252-9.

2. Hendriks JJ, Teunissen CE, de Vries HE, Dijkstra CD. Macrophages and neurodegeneration. Brain Res Brain Res Rev. 2005;48(2):185-95.

3. Barnett MH, Henderson AP, Prineas JW. The macrophage in MS: just a scavenger after all? Pathology and pathogenesis of the acute MS lesion. Mult Scler. 2006;12(2):121-32.

4. Bogie JF, Stinissen P, Hendriks JJ. Macrophage subsets and microglia in multiple sclerosis. Acta Neuropathol. 2014;128(2):191-213.

5. Kotter MR, Zhao C, van Rooijen N, Franklin RJ. Macrophage-depletion induced impairment of experimental CNS remyelination is associated with a reduced oligodendrocyte progenitor cell response and altered growth factor expression. Neurobiol Dis. 2005;18(1):166-75.

6. Schwab ME. Nogo and axon regeneration. Curr Opin Neurobiol. 2004;14(1):118-24.

7. Morell P QR. Characteristic Composition of Myelin. Basic Neurochemistry: Molecular, Cellular and Medical Aspects 6th edition. Philadelphia: Lippincott-Raven; 1999.

8. Saher G, Brugger B, Lappe-Siefke C, Mobius W, Tozawa R, Wehr MC, et al. High cholesterol level is essential for myelin membrane growth. Nat Neurosci. 2005;8(4):468-75.

9. Spady DK, Dietschy JM. Sterol synthesis in vivo in 18 tissues of the squirrel monkey, guinea pig, rabbit, hamster, and rat. J Lipid Res. 1983;24(3):303-15.

10.van de Kraats C, Killestein J, Popescu V, Rijkers E, Vrenken H, Lutjohann D, et al. Oxysterols and cholesterol precursors correlate to magnetic resonance imaging measures of neurodegeneration in multiple sclerosis. Mult Scler. 2014;20(4):412-7.

11.Teunissen CE, Dijkstra CD, Polman CH, Hoogervorst EL, von Bergmann K, Lutjohann D. Decreased levels of the brain specific 24S-hydroxycholesterol and cholesterol precursors in serum of multiple sclerosis patients. Neurosci Lett. 2003;347(3):159-62.

12.Karrenbauer VD, Leoni V, Lim ET, Giovannoni G, Ingle GT, Sastre-Garriga J, et al. Plasma cerebrosterol and magnetic resonance imaging measures in multiple sclerosis. Clin Neurol Neurosurg. 2006;108(5):456-60.

13.Zhao C, Dahlman-Wright K. Liver X receptor in cholesterol metabolism. J Endocrinol. 2010;204(3):233-40.

14.Fan X, Kim HJ, Bouton D, Warner M, Gustafsson JA. Expression of liver X receptor beta is essential for formation of superficial cortical layers and migration of later-born neurons. Proc Natl Acad Sci U S A. 2008;105(36):13445-50.

15.Joseph SB, Bradley MN, Castrillo A, Bruhn KW, Mak PA, Pei L, et al. LXR-dependent gene expression is important for macrophage survival and the innate immune response. Cell. 2004;119(2):299-309. 
16.Joseph SB, Castrillo A, Laffitte BA, Mangelsdorf DJ, Tontonoz P. Reciprocal regulation of inflammation and lipid metabolism by liver $X$ receptors. Nat Med. 2003;9(2):213-9.

17.Ghisletti S, Huang W, Ogawa S, Pascual G, Lin ME, Willson TM, et al. Parallel SUMOylation-dependent pathways mediate gene- and signal-specific transrepression by LXRs and PPARgamma. Mol Cell. 2007;25(1):57-70.

18.Boven LA, Van Meurs M, Van Zwam M, Wierenga-Wolf A, Hintzen RQ, Boot RG, et al. Myelin-laden macrophages are anti-inflammatory, consistent with foam cells in multiple sclerosis. Brain. 2006;129(Pt 2):517-26.

19.Bogie JF, Stinissen P, Hellings N, Hendriks JJ. Myelin-phagocytosing macrophages modulate autoreactive T cell proliferation. J Neuroinflammation. 2011;8:85.

20.Bogie JF, Jorissen W, Mailleux J, Nijland PG, Zelcer N, Vanmierlo T, et al. Myelin alters the inflammatory phenotype of macrophages by activating PPARs. Acta Neuropathol Commun. 2013;1(1):43.

21.Bogie JF, Timmermans S, Huynh-Thu VA, Irrthum A, Smeets HJ, Gustafsson JA, et al. Myelin-derived lipids modulate macrophage activity by liver $X$ receptor activation. PLoS One. 2012;7(9):e44998.

22.Norton WT, Poduslo SE. Myelination in rat brain: changes in myelin composition during brain maturation. J Neurochem. 1973;21(4):759-73.

23.Jansen PJ, Lutjohann D, Abildayeva K, Vanmierlo T, Plosch T, Plat J, et al. Dietary plant sterols accumulate in the brain. Biochim Biophys Acta. 2006;1761(4):445-53.

24.Lutjohann D, Brzezinka A, Barth E, Abramowski D, Staufenbiel M, von Bergmann K, et al. Profile of cholesterol-related sterols in aged amyloid precursor protein transgenic mouse brain. J Lipid Res. 2002;43(7):1078-85.

25.Vanmierlo T, Bogie JF, Mailleux J, Vanmol J, Lutjohann D, Mulder M, et al. Plant sterols: Friend or foe in CNS disorders? Prog Lipid Res. 2015;58:26-39.

26.Yvan-Charvet L, Wang N, Tall AR. Role of HDL, ABCA1, and ABCG1 transporters in cholesterol efflux and immune responses. Arterioscler Thromb Vasc Biol. 2010;30(2):139-43.

27.Mangelsdorf DJ, Evans RM. The RXR heterodimers and orphan receptors. Cell. 1995;83(6):841-50.

28.Janowski BA, Grogan MJ, Jones SA, Wisely GB, Kliewer SA, Corey EJ, et al. Structural requirements of ligands for the oxysterol liver X receptors LXRalpha and LXRbeta. Proc Natl Acad Sci U S A. 1999;96(1):26671.

29.Fu X, Menke JG, Chen Y, Zhou G, MacNaul KL, Wright SD, et al. 27-hydroxycholesterol is an endogenous ligand for liver $X$ receptor in cholesterol-loaded cells. J Biol Chem. 2001;276(42):38378-87.

30.Janowski BA, Willy PJ, Devi TR, Falck JR, Mangelsdorf DJ. An oxysterol signalling pathway mediated by the nuclear receptor LXR alpha. Nature. 1996;383(6602):728-31.

31.Forman BM, Ruan B, Chen J, Schroepfer GJ, Jr., Evans RM. The orphan nuclear receptor LXRalpha is positively and negatively regulated by distinct products of mevalonate metabolism. Proc Natl Acad Sci U S A. 1997;94(20):10588-93.

32.Zhou G, Cummings R, Li Y, Mitra S, Wilkinson HA, Elbrecht A, et al. Nuclear receptors have distinct affinities for coactivators: characterization by fluorescence resonance energy transfer. Mol Endocrinol. 1998;12(10):1594-604.

33. Castrillo A, Joseph SB, Marathe C, Mangelsdorf DJ, Tontonoz P. Liver X receptor-dependent repression of matrix metalloproteinase-9 expression in macrophages. J Biol Chem. 2003;278(12):10443-9. 
34. Meffre D, Shackleford G, Hichor M, Gorgievski V, Tzavara ET, Trousson A, et al. Liver X receptors alpha and beta promote myelination and remyelination in the cerebellum. Proc Natl Acad Sci U S A. 2015;112(24):7587-92.

35. Wang Z, Sadovnick AD, Traboulsee AL, Ross JP, Bernales CQ, Encarnacion M, et al. Nuclear Receptor NR1H3 in Familial Multiple Sclerosis. Neuron. 2016;90(5):948-54. 